\title{
Selected Determinants of Pronunciation Anxiety
}

\author{
MAŁGORZATA BARAN-ŁUCARZ \& JANG HO LEE* \\ University of Wrockaw (Poland) \\ Chung-Ang University (Republic of Korea)
}

Received: 03/05/2020. Accepted: 03/04/2021.

\begin{abstract}
Empirical research shows that language anxiety has a detrimental effect on foreign language learning and its use. Several studies suggest that anxiety related to mastering and using foreign languages is skill-specific. This study examined pronunciation anxiety and attempted to determine its significant correlates. The included factors ranged from learning experiences with native-speaking teachers, previous studying abroad experience, and enjoyment of learning the target language, to willingness to communicate in the target language. A questionnaire was administered to two groups of EFL learners of different majors and different self-perceived levels. Hierarchical multiple regression analyses for both groups showed that willingness to communicate in English was the strongest determinant of pronunciation anxiety, while foreign language enjoyment the second meaningful correlate, but only in the case of the group whose self-assessment of general proficiency in English was lower.
\end{abstract}

KEYWORDS: Foreign language enjoyment; FL learning experience; Language anxiety; Pronunciation anxiety; Willingness to communicate.

\section{INTRODUCTION}

Most previous empirical data have revealed that language anxiety (LA) has a detrimental effect on both foreign language (FL) learning and its use (e.g. Horwitz, Horwitz \& Cope, 1986; Phillips, 1992; Piechurska-Kuciel, 2008). One type of anxiety experienced by FL learners, along with speaking (Phillips, 1992), reading (Saito, Garza \& Horwitz, 1999), writing (Cheng, Horwitz \& Schallert, 1999), and listening (Kim, 2000) anxiety, is pronunciation anxiety (PA)

*Address for correspondence: 84 Heukseok-ro, Dongjak-gu, Seoul, Republic of Korea; e-mail: jangholee@cau.ac.kr 
(Baran-Łucarz, 2014a). As observed by Baran-Łucarz (2014a), PA can affect students' FL learning and use at various proficiency levels. PA seems to determine the learners' eagerness to join in or initiate FL conversations in and outside the classroom (Baran-Łucarz, 2014a). Refraining from speaking hinders FL acquisition, since active involvement in communication is not only the aim of most FL learners, but also a necessary condition to reach it (e.g. Savignon, 2005). Consequently, it seems essential to determine factors that contribute to PA. The identification of these factors can contribute to a better understanding of the construct of PA, and also aid in the search for remedies lowering this apprehension in the FL classroom. To this end, a study aimed at exploring the correlates of PA was conducted, whose results are presented herein.

\section{LITERATURE REVIEW}

\subsection{Towards a definition and model of pronunciation anxiety}

Researchers focusing on FL pronunciation learning and teaching generally agree that these processes often evoke emotions that are incomparable with those accompanying mastering other FL aspects (e.g. Derwing \& Munro, 2015). Pronunciation, unlike other language subskills (grammar, vocabulary), appears to be related to identity (e.g. Lamb, 2004; Setter, 2008). Among the first researchers supporting this idea was Guiora, who explained that pronunciation is "the key to the extent to which the individual is psychologically capable of stepping into a new system of communication" (1972: 144). This can be explained by the fact that "the way we sound" is "one of the basic modes of identification by the self and others" (Guiora, 1972: 144). Similarly, Walker concluded that "whatever accent we have, native speaker or non-native speaker, standard or regional, it is part of our identity" (2011: 14). Consequently, we may hypothesize, consistent with the opinions of many others (e.g. Grazia Busa, 2010; Rindal, 2010), that the FL pronunciation level learners eventually reach is determined to an important extent by their willingness to accept a new FL identity, which evidently differs from their first language (L1) identity. In other words, among the building blocks of this FL identity is the way one sounds, i.e. one's pronunciation.

The idea that FL learning and use are essentially related to changes in identity was supported also by Horwitz et al. (1986: 128), who explained that learners are aware of their "authenticity" being "restricted", and thus fear the danger of their "more limited self" (as opposed to the "true self") being revealed when communicating in a language other than their L1. The fact that FL learning is "inherently stressful to some people" because of "the inability to present oneself authentically and the resulting threat to self-concept" was more recently restressed by Horwitz (2017: 40), who referred to her earlier idea (e.g. 2013) of pink dress anxiety. With this expression, she compares the discomfort caused by limitations of the new FL to wearing clothes and a haircut that one does not accept and feel good in, particularly 
while delivering a public speech. An analogous thought was shared by Baran-Łucarz (2013a) concerning phonetics learning anxiety, suggesting comparability between a learner's pronunciation of an FL and an individual's clothes or make-up: one must feel good and comfortable in it to reach high levels of proficiency.

When pronunciation anxiety is concerned, its working definition and model, forwarded by Baran-Lucarz (2014a), was based on the general concept of anxiety (e.g. Vasa \& Pine, 2004), which arises when one views certain events as threatening to his/her ego (e.g. the necessity to communicate in a FL) and one's ability to cope with the situation as poor (e.g. low self-perceived FL subskills, including pronunciation) (Pekrun, 1992). Being also a type of LA (Horwitz et al., 1986), its conceptualization took into account the three general anxietiescommunication apprehension, test anxiety, and fear of negative evaluation-that LA derived from. Although PA has been suggested as a situation-specific type of anxiety, it was at the same time believed to "have a trait-like nature, being most probably shaped by several individual-learner differences, particularly personality" (Baran-Łucarz, 2014a: 452). The basis for defining PA and suggesting its model was also the fact that students' personal and interpersonal anxieties, comprising self-perceptions and beliefs related to FL learning, proved to be the most influential determinants of LA (e.g. Baran-Łucarz, 2011; Piechurska-Kuciel, 2008; Young, 1991). The fact that worrying about one's FL pronunciation can be an important source of LA was reported several decades ago (e.g. Phillips, 1992) and has been verified again more recently (e.g. Baran-Łucarz, 2014b; MacIntyre, 2017).

Based on observations of and interviews with EFL learners (e.g. Baran-Łucarz, 2013a, $2013 \mathrm{~b}$ ), the vast body of information on LA and its sources, and the typical dimensions of any type of anxiety (e.g. Piechurska-Kuciel, 2008; Vasa \& Pine, 2004), PA has been defined as a:

[...] a multidimensional construct referring to the feeling of apprehension and worry experienced by non-native speakers in oral-communicative situations [...] deriving from their negative/low self-perceptions, beliefs and fears related specifically to pronunciation (BaranŁucarz, 2017: 109).

PA is thus a multifaceted concept, composed of four subcomponents (Baran-Łucarz, 2014a, 2016, 2017). These, though independent, interact with each other dynamically (see Baran-Łucarz, 2016). The building blocks of PA include the following: (1) fear of negative evaluation from potential interlocutors, classmates, or teachers, specifically due to pronunciation problems; (2) pronunciation self-efficacy and self-assessment based on perceptions of one's pronunciation strengths and weaknesses, particularly in comparison to those of other classmates or interlocutors; (3) pronunciation self-image (i.e., the conception of one's aural and visual appearance while speaking the target language (TL) and readiness to accept this image); and (4) beliefs concerning the difficulty of the TL phonological system for 
speakers of a particular L1, beliefs about the importance of pronunciation for communication, and attitudes towards the general sound of the TL pronunciation.

PA was found to indicate a shared variance of 51.8\% with LA (Baran-Łucarz, 2016), which suggests that although the two are strongly linked, "PA has its own specific and unique nature, different from LA" (Baran-Łucarz, 2017: 110). Despite the fact that the PA model can refer to both formal and informal contexts, the extent to which each of the sub-components determines the final shape of the construct in these two settings may vary due to their unique characteristics (Baran-Łucarz, 2017).

\subsection{Potential determinants of pronunciation anxiety}

On the basis of literature analysis, we selected several potential external and internal factors that may affect PA: learning experiences with native-speaking teachers and study abroad experiences (external factors), and enjoyment of TL learning and willingness to communicate (WTC) in the TL (internal factors). A brief introduction of the potential PA correlates and our rationale for choosing them are offered below.

\subsubsection{Learning experiences with native-speaking teachers}

The first factor selected as a possible external determinant of PA was the amount of previous TL learning experience with native-speaking teachers. In Levis, Sonsaat, Link, and Barriuso's (2016) study, two groups of students-one taught by a native English-speaking teacher (NEST) and the other by a non-native English-speaking teacher (NNEST)-were offered the same type and amount of pronunciation instruction for seven weeks. The quantitative results showed that the progress made by participants in their level of accentedness and comprehensibility did not vary significantly between the groups. However, the qualitative data revealed that most of the participants preferred "having a native teacher for a pronunciation class" (Levis et al., 2016: 915). As the researchers suggested, attitudes towards NESTs regarding TL pronunciation teaching and learning may be rooted in the belief "that having an NEST would somehow result in greater improvement by 'catching' pronunciation in the same way one catches a cold, through experience alone" (Levis et al., 2016: 915). Although this may not necessarily be true, such a belief may raise the self-evaluation of TL pronunciation of those taught by native-speaking teachers and, in turn, reduce PA levels. In addition, some learners have been found to perceive NESTs as friendlier and as those who establish more relaxed learning environments than NNESTs (Tang \& Johnson, 1993).

On the other hand, some participants of Ma's (2012) study acknowledged feeling anxious during FL classes with NESTs. Moreover, findings of other observations (Lipovsky \& Mahboob, 2010; Ma, 2012) have suggested that FL teachers sharing the students' L1 were more empathetic to learners' needs, more capable of setting realistic goals, and more sensitive 
to difficulties learners may encounter than NESTs. These features may help keep classroom anxiety minimal.

Since PA is largely derived from students' perceptions and beliefs, we assumed this factor (i.e. being taught by a TL native-speaking teacher), which seems to shape the selfperceptions of students' FL skills and evoke a ray of different emotions, may be a determinant of PA.

\subsubsection{Study abroad experience}

Study abroad (SA) researchers have examined the relationship between learners' SA experience and their level of anxiety in FL learning. Thompson and Lee (2014) found that having prior SA experience was negatively correlated to FL classroom anxiety (FLCA) among undergraduate Korean EFL learners, as measured by the FLCA Scale (Horwitz et al., 1986). More evidence was provided by Allen and Herron (2003), who observed that the language anxiety of 25 college students who participated in a six-week French SA program decreased significantly. More recently, Lee (2018) examined the effects of short-term SA experience on variables such as WTC and FLCA with Korean college learners. The results again showed that the students' level of FLCA dropped significantly after five weeks of SA experience, whereas their WTC increased.

Besides the results of earlier studies mentioned above, there are also a few assumptions related to PA specifically that have encouraged us to examine the predictive strength of SA experience for PA. First of all, studying abroad requires constant TL use for authentic communicative purposes (usually despite language deficiencies), typically free from language correction. When successful, this might not only reduce general speaking apprehension but also decrease the fear of one's performance being negatively evaluated by interlocutors due to potential mispronunciations. This, in turn, may lead to higher pronunciation self-efficacy and self-assessment. Moreover, systematic effective TL use might also help speakers accept their changing TL pronunciation self-images, one of the building blocks of PA.

\subsubsection{Foreign language enjoyment}

The construct of foreign language enjoyment (FLE) has been forwarded by Dewaele and MacIntyre (2014), as a positive emotion experienced by students in the FL classroom due to positive classroom dynamics, shaped by the teacher, learners, the relationships among them, fun and laughter, and the self of security and confidence. FLE and LA appear to share a theoretical link, as both focus on the 'affect' and 'emotion' of language learners (Dewaele, Witney, Saito \& Dewaele, 2018). Having in mind that FLE are positive emotions, it is worth referring to Dewaele and Alfawzan, who state as follows: "Positive emotions can ... drive out negative arousal, which is crucial because negative emotions cause a narrowing of focus and limit the potential language input", and they can "have longer-term effects outside the 
classroom as they can make students more resilient and hardy during difficult times" (2018: 26).

In Dewaele and MacIntyre's (2014) study, a negative correlation of moderate strength was found between FLE and FLCA. Based on this finding, Dewaele and MacIntyre suggested that "these two dimensions are related but that enjoyment and anxiety appear to be independent emotions, and not opposite ends of the same dimension" (2014: 261). In Dewaele and Dewaele's (2017) study with FL pupils in UK public schools, though, the relationship between FL classroom anxiety (FLCA) and FLE was only significant for a certain age group, possibly because FLE was found to fluctuate over time, whereas FLCA largely remained unchanged. Since PA is so heavily loaded with emotions, it can be assumed to be shaped by the enjoyment students experience or have experienced learning the TL.

\subsubsection{L2 willingness to communicate}

The final variable taken into account as a potential correlate of PA is willingness to communicate in a second language (henceforth, L2 WTC) ${ }^{1}$, defined as "a readiness to enter into discourse at a particular time with a specific person or persons, using [an] L2" (MacIntyre, Clément, Dörnyei \& Noels, 1998: 547). In their heuristic pyramid model, MacIntyre et al. (1998) present L2 WTC as a complex, multifaceted construct composed of six layers, three representing immediate/situational (Layers I-III) factors, and three-more distant but stable antecedents (Layers IV-VI). Several researchers who explored the nature of the construct from a macro-perspective lent support to the aforementioned model, showing that anxiety (e.g. MacIntyre \& Legatto, 2011), intrinsic and extrinsic motivation (e.g. Hashimoto, 2002), beliefs (e.g. Peng, 2007), and attitudes related to FL learning (e.g. Baker \& MacIntyre, 2000) are significant determinants of L2 WTC and its use. Thanks to a more recent trend of examining the construct from a classroom (micro) perspective, the importance of the more immediate situational antecedents has been verified, such as the significance of the learning situation and student's subjective perception of it (Zhang, Beckmann \& Beckmann, 2018), familiarity with the interlocutors (e.g. Cao \& Philp, 2006), or class size (Cao \& Philp, 2006; Khazaei, Zahed \& Ketabi, 2012). Some (e.g. Mystkowska-Wiertelak \& Pawlak, 2017) have managed to combine the macro- and micro-perspectives, showing that despite individuals revealing certain stable dispositions of L2 WTC, their WTC may fluctuate over time depending on learning conditions.

It is important to add that L2 WTC is culture-dependent, due to it being related to the concepts of ethnolinguistic vitality and subjective group vitality (Giles, Bourhis \& Taylor, 1977), and it being determined by "cultures of learning and communication" (Peng, 2014: 29), which are unique for every country. Moreover, it is also self-confidence that has been found to be rooted in the culture one is brought up in. This seems particularly important in the light of Gregersen and MacIntyre's claim that "researchers have consistently found that high FL anxiety and low TL self-confidence are the greatest predictors of a learner's unwillingness to 
communicate" (2014: 221). This has led Baran-Łucarz (2014a) to examine the relationship between L2 WTC and specifically PA, among Polish university students, and the results pointed to the high correlation between these two constructs $(r=-.60, p<.0005)$. Although correlation analysis does not show directionality, responses gathered via semi-structured interviews suggested that PA shaped participants' L2 WTC. It is, however, also possible that L2 WTC determines learners' PA. Students who are more eager to speak an L2, particularly outside the classroom, may expose themselves to more input, and offer themselves more practice in perception and production. Though this may not guarantee higher levels in pronunciation (there are many other determinants of progress in intelligibility and pronunciation accuracy such as formal instruction, students' aptitude, concern for pronunciation, and phonetic awareness (e.g. Derwing \& Munro, 2015)), there are other potential benefits from more frequent contact with the authentic TL. Learners with higher levels of WTC, having experienced effective communicative situations, may reveal more realistic beliefs about the importance of pronunciation in communication, realizing for example that even accented pronunciation can be intelligible (e.g. Munro \& Derwing, 1995). Moreover, they may see more easily that all non-native speakers make mistakes, also in pronunciation, and are rarely evaluated by their interlocutors, irrespective of their L1. This, in turn, might help decrease the fear of being negatively viewed by others, and positively change the pronunciation self-assessment, self-efficacy, and self-image, all of which have been suggested as antecedents of PA.

Based on the review of LA and PA research, a study was conducted to find the answers to the following research questions:

RQ1: Which of the selected variables - TL experience with native-speaking teachers, study abroad experience, foreign language enjoyment, and L2 WTC - are the best determinants of PA in the FL classroom?

RQ2: Do the selected PA determinants vary between two groups of English learners of different majors and self-perceived English proficiency levels?

\section{METHOD}

\subsection{Participants}

The participants were from two different undergraduate institutions in Seoul, Republic of Korea in 2017. Based on Dewaele and Dewaele's (2017) research, we included two learner groups of different majors and self-perceived English proficiency levels. One group majored in English education ( $n=86$ ) (EE Group, henceforth), and the other majored in tourism English, aiming to be either flight attendants or employees in the hospitality industry $(n=67)$ (TE Group, henceforth). Fifty-eight participants in the EE Group and all the TE Group participants were female. Both groups were registered in mandatory English conversation 
courses. The participants in each institution were taught by different English instructors, respectively. On average, the EE Group participants were 21.9 years old, and the TE group participants were 20.7 years old. Their self-perceived competence in English differed significantly, with the EE Group assessing their English skills higher than the TE Group $(t=$ 8.87, $p$ <.001) (EE Group: $M=6.79, S D=1.18, n=84$; TE Group: $M=4.62, S D=1.68, n=$ 65 ), as measured by averaging their responses to four 10-point scale items regarding different language skills (i.e. listening, speaking, reading, and writing). Regarding their self-perceived competence in speaking only, the two groups also differed significantly $(t=6.44, p<.001)$ (EE Group: $M=5.86, S D=1.80, n=84$; TE Group: $M=3.95, S D=1.78, n=65$ ). Further differences between the groups are presented in Descriptive statistics in the Results.

\subsection{Instruments}

A questionnaire consisting of three sub-sections was used for data collection. The first section focused on participants' background, and the second and third concerned PA and its potential correlates. Most of the questionnaire items were adapted from previous studies (Baran-Łucarz, 2017; Dewaele \& MacIntyre, 2014; Peng \& Woodrow, 2010), translated into the participants' L1 (Korean), as some of the English terms in the original questionnaires were judged to be difficult to understand for the target students by the authors. The initial questionnaire was further piloted with 17 undergraduate EFL learners similar in profile to the participants of the main study. They were asked to examine the questionnaire items for comprehensibility and relevance. The introduction to the second and third sections and their internal consistency (i.e. Cronbach's alpha) are described below.

\subsubsection{Measure of pronunciation anxiety}

To diagnose participants' PA level, an adapted version of the Measure of Pronunciation Anxiety (MPA) (Baran-Łucarz, 2017) was applied. Among the 50 Likert-scale items from the original MPA, ten concerning participants' anxiety level regarding TL communication outside the classroom were not used, as the pilot study suggested that our target students rarely used English in natural contexts. Additionally, based on the pilot study, four questionnaire items were removed because of irrelevance to the target students. For example, it was found in the pilot study that none of its participants stated that they would imitate English actors/singers (\#19. in the original questionnaire) or rehearse English speaking at home (\#2. in the original questionnaire).

All items used a 6-point Likert scale, with higher scores indicating higher levels of PA. Some items denoted no/low PA (e.g. 'I look natural speaking English'), so participants' responses to those items were reverse-coded. Based on Baran-Łucarz (2017), the questionnaire items, relevant to each sub-component of PA, were merged into one variable. During this process, two items (\#16. I do/would not mind pronouncing English sounds and/or words with 
Korean accents, \#28. English is like music to me) were removed, as they negatively affected sub-component reliability. Next, the internal consistency of the whole questionnaire and its seven sub-components were examined and the sub-scale correlations were verified (see Table $1)$. 
Table 1. Internal Consistency (Cronbach's alpha) of the Sub-components of the MPA and Correlations among Them (Note: *p<.05.**p<.01.)

\begin{tabular}{|c|c|c|c|c|c|c|c|c|}
\hline Variables (internal consistency) & 1. & 2. & 3. & 4. & 5. & 6. & 7. & 8. \\
\hline $\begin{array}{l}\text { 1. General oral performance } \\
\text { apprehension in English }(\alpha=.76)\end{array}$ & 1 & $.43 * *$ & $.45^{* *}$ & $.60 * *$ & $.40 * *$ & .03 & $.36^{* *}$ & $71 * *$ \\
\hline $\begin{array}{l}\text { 2. Pronunciation self-efficacy and self- } \\
\text { assessment }(\alpha=.78)\end{array}$ & & 1 & $.79 * *$ & $.38 * *$ & $.39 * *$ & .03 & $.32 * *$ & $70 * *$ \\
\hline 3. Pronunciation self-image $(\alpha=.85)$ & & & 1 & $.43 * *$ & $.50 * *$ & .04 & $.36^{* *}$ & $78^{* *}$ \\
\hline 4. Fear of negative evaluation $(\alpha=.90)$ & & & & 1 & $.54 * *$ & .04 & $.34 * *$ & $75^{* *}$ \\
\hline $\begin{array}{l}\text { 5. Beliefs about the nature/sound of } \\
\text { English }(\alpha=.71)\end{array}$ & & & & & 1 & .06 & $.36^{* *}$ & $73 * *$ \\
\hline $\begin{array}{l}\text { 6. Beliefs about the importance of } \\
\text { pronunciation for communication }(\alpha \\
=.50)\end{array}$ & & & & & & 1 & -.01 & $21^{*}$ \\
\hline $\begin{array}{l}\text { 7. Beliefs about difficulties with } \\
\text { learning English pronunciation ( } \alpha \\
=.78)\end{array}$ & & & & & & & 1 & $56^{* *}$ \\
\hline 8. PA total (all items merged) $(\alpha=.92)$ & & & & & & & & 1 \\
\hline
\end{tabular}

Since one sub-component-beliefs about the importance of pronunciation for communication-did not correlate with the remaining scales and only weakly with PA total, it was not considered in further analyses, which reduced the PA sub-components to six. Removing this sub-scale raised the MPA internal consistency to $\alpha=.9$

(C) Servicio de Publicaciones. Universidad de Murcia. All rights reserved. IJES, vol. 21 (1), 2021, pp. 93-113 


\subsubsection{Measures of potential PA determinants}

In the third section, the participants were asked about the number of semesters they had been taught by native English-speaking teachers, if any (i.e. NEST experience). Then, they were to specify the amount of time (months) they had spent studying abroad in Englishspeaking countries (i.e. SA experience).

For foreign language enjoyment, a ten-item questionnaire developed by Dewaele and MacIntyre (2014) was piloted with the aforementioned learners. Like PA, it used a 6-point Likert scale, ranging from ' $1=$ strongly disagree' to ' $6=$ strongly agree'. One item from the original scale (i.e. 'I'm a worthy member of the foreign language class') was removed, as it was deemed semantically awkward by the pilot participants. The nine-item questionnaire had a high level of internal consistency $(\alpha=.91)$.

Finally, to examine L2 WTC, ten 6-point Likert scale items were adapted from Peng and Woodrow (2010). All questionnaire items concerned L2 WTC in a variety of FL classroom situations, ranging from asking peers about the meaning of an unfamiliar English word to giving a short speech to the class. For each item, the participants were to mark one option from 1 (very unwilling to communicate in English in the target situation) to 6 (very willing to communicate in English in the target situation). The questionnaire items remained intact after the pilot study. Based on the high internal consistency $(\alpha=.95)$, all items were merged to create the $L 2$ WTC variable.

\subsection{Data collection and analysis}

The 15-20-minute questionnaire was distributed among the participants during a midsemester regular English class. They were informed that the study concerned EFL learners' perceptions of their English learning and that their responses would be treated anonymously and would not affect their grades. The participants were also told that they were free to opt out; however, none of them decided to do so.

When data analysis is concerned, several steps were taken. After verifying the internal consistencies of the instruments and their subcomponents, descriptive statistics of the dependent and independent variables were computed. Then, simple regressions were calculated separately for each of the potential correlates of PA. There were two reasons for such a decision. First of all, as reported above, several earlier studies have suggested that the factors selected by us are related significantly to language anxiety. However, in previous research the predictive strength of each of these variables were tested separately. Moreover, in earlier studies, it was general LA and not PA that functioned as the dependent variable. Consequently, we recognized the necessity to verify whether the factors selected by us are at all relevant in our sample. Secondly, the results of the simple regressions were to show us the 
order in which the variables should be entered into the hierarchical regression models. Once the assumptions underlying the regression models were verified, two hierarchical multiple regression models were run, with one for the EE Group and the other for the TE Group.

\section{RESULTS}

\subsection{Descriptive statistics}

Table 2 shows that both groups reported to have had on average over six semesters of learning experience with native English-speaking teachers, with the EE Group revealing a slightly longer experience. However, it is important to stress that the groups had different amounts of SA experience. While the EE Group had on average 6.5 months of SA experience, with a wide range of SA experience $(S D=12.51)$, the TE Group showed high homogeneity in revealing little SA experience. When FLE is concerned, the mean values signify a comparable and moderate level of FLE in both groups. Regarding willingness to communicate in English in the classroom, the EE Group was more eager to talk than the TE Group. Finally, both groups had a moderate level of PA, with the TE showing slightly more apprehension related to pronunciation.

Table 2. Descriptive Statistics of the Target Variables.

\begin{tabular}{lcccccc}
\hline & \multicolumn{3}{c}{ EE Group $(n=86)$} & \multicolumn{3}{c}{ TE Group $(n=67)$} \\
\hline & Mean & $\begin{array}{c}\text { Standard } \\
\text { deviation }\end{array}$ & $\begin{array}{c}\text { Standard } \\
\text { error }\end{array}$ & Mean & $\begin{array}{c}\text { Standard } \\
\text { deviation }\end{array}$ & $\begin{array}{c}\text { Standard } \\
\text { error }\end{array}$ \\
\hline $\begin{array}{l}\text { NEST experience } \\
\text { (in semesters) }\end{array}$ & 7.50 & 5.11 & .55 & 6.42 & 5.15 & .63 \\
$\begin{array}{l}\text { SA experience } \\
\text { (in months) }\end{array}$ & 6.51 & 12.51 & 1.35 & .24 & .87 & .11 \\
FLE & 4.49 & .71 & .08 & 4.18 & .93 & .11 \\
L2 WTC & 4.45 & .98 & .11 & 3.52 & 1.03 & .13 \\
PA & 3.20 & .73 & .08 & 3.41 & .69 & .08 \\
\hline
\end{tabular}

\subsection{Simple regression and multiple regression analyses}

As stated earlier, the final decision on the selection of correlates and their order of entry into the hierarchical regression models was made on the basis of results of simple linear regressions computed first for each of the variables separately, with the criterion variable being PA. The linear regression assumptions were verified (assumptions of linearity, normality, 
independence, homoscedasticity, and of absence of collinearity) and our data were found not to violate any of them. As can be seen in Table 3, the outcomes of the linear regressions revealed that, in the case of the EE group, L2 WTC, FLE, and SA experience were significant determinants of PA, with L2 WTC and SA experience showing the largest and smallest $R^{2}$, respectively. When the TE is concerned, only L2 WTC and FLE were significant determinants of PA, with L2 WTC showing a higher $R^{2}$. Consequently, a decision was made to compute hierarchical regressions for EE and TE, with L2 WTC introduced as the first variable and FLE as the second variable. SA experience was additionally introduced as the third variable, but for the EE group only. Below, the hierarchical regression models for each group are presented. As previously, all the assumptions underlying regression analysis were met (the Q-Q plots and analyses of kurtoses and skewedness showed that the residuals were normally distributed; all the VIF values were below 1.8; there were no influential cases-Cook's distances and leverage values were in the range $0-0.25$; the Durbin Watson values were between 0 and 2).

Table 3. Results of Linear Regressions.

\begin{tabular}{llccccc}
\hline Group & Variables & $B$ & $S E$ & $\beta$ & $p$ & $R^{2}$ \\
\hline \multirow{5}{*}{ EE Group } & L2 WTC & -.47 & .06 & -.63 & .00 & .40 \\
& FLE & -.51 & .10 & -.50 & .00 & .25 \\
& SA experience & -.02 & .01 & -.27 & .01 & .07 \\
& NEST experience & -.02 & .02 & -.15 & .17 & .02 \\
\hline \multirow{5}{*}{ TE Group } & L2 WTC & -.39 & .07 & -.58 & .00 & .33 \\
& FLE & -.38 & .08 & -.52 & .00 & .27 \\
& SA experience & -.07 & .10 & -.09 & .48 & .01 \\
& NEST experience & -.03 & .02 & -.20 & .11 & .04 \\
\hline
\end{tabular}

\subsubsection{The EE Group}

The results for the more advanced EE Group, with PA as a criterion variable, are presented in Table 4.

As shown in Table 4, the final model, which included L2 WTC, FLE and SA experience as determinants of PA revealed that together they accounted for about $47 \%$ of the variance in PA $\left(R^{2}=.47, F(3,82)=24.45, p<.001\right)$. In this final step, L2 WTC was the only significant correlate for this group, whereas FLE almost reached a level of statistical significance $(p=$ $.06)$. Both of these variables were negatively related to PA, with the standardized beta value $(\beta)$ for $\mathrm{L} 2 \mathrm{WTC}=-.53$ and for FLE $=-.19$. 
Table 4. EE Group: Multiple Regression Analysis Predicting PA $(* * * p<.001)$.

\begin{tabular}{llcccccc}
\hline & Variables & $B$ & $S E$ & $\beta$ & $p$ & $R^{2}$ & $\Delta R^{2}$ \\
\hline Step 1 & Constant & 5.31 & .29 & & & $.40^{* * *}$ & - \\
& L2 WTC & -.47 & .06 & -.63 & .00 & & \\
\hline Step 2 & Constant & 6.10 & .39 & & & & \\
& L2 WTC & -.39 & .07 & -.51 & .00 & $.45^{* * *}$ & .05 \\
& FLE & -.26 & .09 & -.26 & .00 & & \\
\hline Step 3 & Constant & 5.91 & .41 & & & & .02 \\
& L2 WTC & -.40 & .07 & -.53 & .00 & $.47^{* * *}$ & \\
& FLE & -.20 & .10 & -.19 & .06 & & \\
& SA experience & -.01 & .01 & -.15 & .09 & & \\
\hline
\end{tabular}

\subsubsection{The TE Group}

The hierarchical multiple regression model was run again for the TE Group. The results of this analysis, with PA as a criterion variable, are presented in Table 5.

Table 5. TE Group: Multiple Regression Analysis Predicting PA (*** $p<.001)$.

\begin{tabular}{llcccccc}
\hline & Variables & $B$ & $S E$ & $\beta$ & $p$ & $R^{2}$ & $\Delta R^{2}$ \\
\hline Step 1 & Constant & 4.78 & .25 & & & $.33^{* * *}$ & - \\
& L2 WTC & -.39 & .07 & -.58 & .00 & & \\
\hline Step 2 & Constant & 5.21 & .32 & & & & \\
& L2 WTC & -.28 & .08 & -.42 & .001 & $.37^{* * *}$ & .04 \\
& FLE & -.19 & .09 & -.26 & .04 & & \\
\hline
\end{tabular}

In the final model, the two factors appeared to be statistically significant correlates of PA and together they accounted for about $37 \%$ of variance in PA. Both of the variables were negatively related to PA, with L2 WTC being evidently a stronger determinant than FLE (L2 $\operatorname{WTC}(\beta)=-.42 ; \operatorname{FLE}(\beta)=-.26)$.

\section{DISCUSSION}

The aim of the reported study was to examine which of the selected variables - TL experience with native-speaking teachers, study abroad experience, foreign language enjoyment (FLE), or willingness to communicate in a second language (L2 WTC) - are the most significant determinants of PA in the FL classroom and whether they vary depending on the majors of the participants and their self-assessed proficiency level. According to the results of simple linear regressions computed for each of the potential correlates of pronunciation anxiety (PA) separately for the two participant groups, native English-speaking teachers 
(NEST) experience was not found to be a significant determinant of PA experienced in formal contexts. As presented in the theory, there were arguments for assuming NEST to be a possible PA predictor, with previous studies on NEST experience raising (Lipovsky \& Mahboob, 2010; Ma, 2012) or lowering language anxiety (Tang \& Johnson, 1993), or others reporting the belief of many students in the ability to 'catch' native-like pronunciation from NESTs (Levis et al., 2016). However, it needs to be stressed that in this study it is unknown whether the teachers (native or non-native) explicitly focused on pronunciation, how they practiced this aspect with their students, or how they handled mispronunciations. It is possible that these classroom factors are more crucial in shaping PA than whether the teacher is a native speaker of the TL or not. It would be also interesting to observe if and how a combination of these two factors (L1 of the teacher and type of explicit pronunciation instruction) would determine the level of PA. Further studies are necessary to examine this matter more thoroughly.

When study abroad experience is concerned, the results of simple linear regressions suggested that it may be considered a determinant of PA, but only in the case of the EE Group that majored in English education and considered their English level higher. However, when introduced into the hierarchical regression model, SA experience did not prove to be a significant correlate of PA, though it added an incremental $R^{2}$ of $2 \%$. Our choice of SA as a potential correlate of PA was based on previous studies in which language anxiety (LA) was one of the dependent variables and on a few assumptions related to the possible influence of SA experience on PA, reported in the theoretical subsection of the paper (Allen \& Herron, 2003; Lee, 2018; Thompson \& Lee, 2014). In our sample, however, SA experience evidently failed to be a significant correlate of PA. This seems to lend support to the idea that PA and LA are linked but independent concepts (Baran-Łucarz, 2017). It also implies that our assumptions were not met, suggesting that PA is indeed a complex construct. Further research on the nature of PA has shown that it is culturally-bound (Baran-Łucarz, in press). This seems to be supported also by the data reported herein, gathered among Korean EFL learners. In this sample, one of the suggested components of PA (i.e. the beliefs related to the importance of pronunciation for communication) functioned differently than in the case of the earlier studies conducted among Poles (e.g. Baran-Łucarz, 2014a, 2014b, 2016). It is possible that the influence of exposure to the TL in naturalistic contexts on PA will vary from culture to culture. For learners representing one culture, effective communication might positively shape their pronunciation images, self-efficacy and self-assessment, while for others this may not necessarily be the case. It must also be added that no qualitative data were gathered that would lend support to the assumption that the SA experience of the participants involved in this study was indeed positive.

The next factor that we suggested as a potential correlate of PA was FLE. The hierarchical model calculated for TE Group with a lower self-assessment of English proficiency showed that FLE was the second strongest determinant of PA. Though PA differs from LA, these results are consistent with the outcomes obtained by Dewaele and MacIntyre 
(2014) and Dewaele and Dewaele (2017), who found negative correlations between FL anxiety and enjoyment of moderate and weak strength, respectively. Taking into account the fact that pronunciation and its learning are emotional (e.g. Derwing \& Munro, 2015) and that the fear of negative evaluation caused by pronunciation is among the central subcomponents of PA (see Table 1), we can hypothesize that fun, laughter, good rapport among students, a positive classroom atmosphere, and feeling of confidence and security during TL lessons can help lower participants' PA level. While the importance of the relations among students, the positive classroom atmosphere, and the worry of classmates' negative views have already been observed (e.g. Baran-Łucarz, 2013b, 2014a), studies are needed to verify the effects of introducing fun and laughter on PA. When the inclusion of FLE into the regression model for the more advanced self-assessed EE Group is concerned, the outcomes are yet different. This time, FLE did not appear to be a determinant of PA, though it was at the boarder of statistical significance $(p=.06)$.

As the final hierarchical regression models for both the EE and the TE showed, the strongest determinant of PA was L2 WTC. It seems worth referring to the outcomes of an earlier mixed-method study (Baran-Łucarz, 2014a) conducted with Polish undergraduate EFL students, which aimed to verify the link between PA and L2 WTC. The results of that study a high negative correlation between these variables and significantly lower levels of WTC among high PA participants relative to those of low PA students - lend support to this study's outcomes. Baran-Łucarz's (2014a) quantitative results were further complemented by her qualitative data. Among explanations for reluctance to speak English in the FL classroom was often anxiety related to uncertainties about pronunciation and self-perceived poor pronunciation, which led to the fear of being negatively viewed by others. Based on these results, it appears that these variables work in tandem, with PA influencing WTC and vice versa.

As reported above, the two groups showed some important difference in the hierarchical regression models, in terms of the relative influences of L2 WTC and FLE on PA. A closer look at FLE's standardized beta $(\beta)$ value (-.19 for the EE Group and -.26 for the TE Group) in relation to L2 WTC's standardized beta value (-.53 for the EE Group and -.42 for the TE Group) implies that the role of FLE may be particularly vital in the case of learners who consider their FL proficiency level lower. This finding provides an important pedagogical implication: L2 teachers working in particular with students perceiving their skills low should try to create an enjoyable L2 learning environment, potentially decreasing their PA and making them more willing to talk. It is also important to draw attention to the fact that while the final model of the EE Group, with L2 WTC being the only statistically significant determinant, accounted for as much as $47 \%$ of variance of PA, the two factor model of the TE Group accounted for much less (i.e. 37\% of variance in PA). This implies that more space is left for the influence of other factors on the level of PA in the case of the TE Group. 


\section{CONCLUSION}

The data in our study suggest that among the variables we chose, including the influence of learning experiences with native-speaking teachers, previous experience studying abroad, and FLE, L2 WTC is the strongest and most significant determinant of PA in the FL classroom. This appeared true irrespective of participants' self-perceived proficiency level. L2 WTC is a complex, multifaceted construct, composed of several antecedents (MacIntyre et al., 1998). While some are more internal and enduring variables (e.g. personality), insensitive to educational approaches and teaching styles, others can be more or less directly shaped by the teacher (e.g. the desire to speak with a particular person about a particular matter, or the knowledge of the topic to be discussed). Some studies imply that interesting topics (Tóth, 2017) and the degree of acquaintance with the interlocutor (Baran-Łucarz, 2014a) can also determine the FL speaking anxiety level. Furthermore, FLE may be another important variable in terms of learners' PA level, and its relationship with L2 WTC and PA should be further examined.

The present study should be considered a first step in the pursuit of a better understanding of the sources of PA. Future research should explore which subcomponents of WTC have the greatest influence on PA. We may also hypothesize that it is a unique and dynamic combination of some of these antecedents that shape PA, rather than one or a few of them. Moreover, it must be emphasized that although L2 WTC has been identified as the most important factor determining PA from among those examined in the multiple regression models, there is still a large amount of variance not accounted for by the selected correlates of this study. What seems worth examining is the role of external factors, such as pronunciation formal instruction (e.g. the use of different approaches and techniques) and of other internal factors such as the students' motivation or various dimensions of cognitive style (field dependence/independence, tolerance of ambiguity) in shaping PA, which awaits future research. Additionally, the students' learning experience prior to their undergraduate study could also be considered in further research.

\section{NOTES}

${ }^{1}$ Although the study reported in our paper involved participants for whom English was a foreign language, rather than a second language, the term L2 WTC is used because it is more common in SLA and it embraces both foreign language and second language WTC.

\section{REFERENCES}

Allen, H. W. \& Herron, C.A. (2003). A mixed-methodology investigation of the linguistic and affective outcomes of summer study abroad. Foreign Language Annals, 36(3), 370-385. 
Baker, S. C. \& MacIntyre, P. D. (2000). The role of gender and immersion in communication and second language orientations. Language Learning, 50, 311-341.

Baran-Łucarz, M. (2011). The relationship between language anxiety and the actual and perceived levels of FL pronunciation. Studies in Second Language Learning and Teaching, 1(4), 491-514.

Baran-Łucarz, M. (2013a). Phonetics learning anxiety - results of a preliminary study. Research in Language, 11(1), 57-79.

Baran-Łucarz, M. (2013b). Anxiety in a FL pronunciation course. In J. Przedlacka, J. Maidment \& M. Ashby (Eds.), Proceedings of PTLC2013, Papers from the Phonetics Teaching and Learning Conference (pp. 19-22). London: PTLC.

Baran-Łucarz, M. (2014a). The link between pronunciation anxiety and willingness to communicate in the foreign language classroom: The Polish EFL context. Canadian Modern Language Review, $70(4), 445-473$.

Baran-Łucarz, M. (2014b). Researching phonetics learning anxiety: A qualitative approach. In D. Gabryś-Barker \& A. Wojtaszek (Eds.), Studying Second Language Acquisition from a Qualitative Perspective (pp. 160-174). Switzerland: Springer International Publishing.

Baran-Łucarz, M. (2016). Conceptualizing and measuring the construct of pronunciation anxiety: Results of a pilot study. In M. Pawlak (Ed.), Classroom-oriented Research: Reconciling Theory and Practice (pp. 39-56). Heidelberg \& New York: Springer.

Baran-Łucarz, M. (2017). FL pronunciation anxiety and motivation: Results of a preliminary mixedmethod study. In E. Szymańska-Czaplak, M. Szyszka \& E. Kuciel-Piechurska (Eds.), At the Crossroads: Challenges in FL Learning (pp. 107-133). Heidelberg \& New York: Springer.

Baran- $€$ ucarz, M. (in press). The mediating effect of culture on the relationship between FL selfassessment and L2 willingness to communicate: The Polish and Italian EFL context. In N. Zarrinabadi \& M. Pawlak (Eds.), New Perspectives on Willingness to Communicate in a Second Language. Heidelberg \& New York: Springer.

Cao, Y. Q. \& Philp, J. (2006). Interactional context and willingness to communicate: A comparison of behavior in whole class, group and dyadic interaction. System, 34, 480-493.

Cheng, Y.-S., Horwitz, E. K. \& Schallert, D. L. (1999). Language anxiety: Differentiating writing and speaking components. Language Learning, 49, 417-446.

Derwing, T. M. \& Munro, M. J. (2015). Pronunciation Fundamentals: Evidence-based Perspectives for L2 Teaching and Research (Vol. 42). Amsterdam: John Benjamins.

Dewaele, J.-M. \& Alfawzan, M. (2018). Does the effect of enjoyment outweigh that of anxiety in foreign language performance? Studies in Second Language Learning and Teaching, 8(1), 2145.

Dewaele, J.-M. \& Dewaele, L. (2017). The dynamic interactions in foreign language classroom anxiety and foreign language enjoyment of pupils aged 12 to 18. A pseudo-longitudinal investigation. Journal of the European Second Language Association, 1(1), 12-22.

(C) Servicio de Publicaciones. Universidad de Murcia. All rights reserved. IJES, vol. 21(1), 2021, pp. 93-113 Print ISSN: 1578-7044; Online ISSN: 1989-6131 
Dewaele, J.-M. \& MacIntyre, P. D. (2014). The two faces of Janus? Anxiety and enjoyment in the foreign language classroom. Studies in Second Language Learning and Teaching, 4(2), 237274.

Dewaele, J.-M., Witney, J., Saito, K. \& Dewaele, L. (2018). Foreign language enjoyment and anxiety: The effect of teacher and learner variables. Language Teaching Research, 22(6), 676-697.

Giles, H., Bourhis, R. Y. \& Taylor, D. M. (1977). Toward a theory of Language in Ethnic group relations. In H. Giles (Ed.), Language, Ethnicity, and Intergroup Relations (pp. 307-348). London: Academic Press.

Grazia Busa, M. (2010). Effects of L1 on L2 pronunciation: Italian prosody in English. Lingusitic Insights - Studies in Language and Communication, 69, 207-228.

Gregersen, T. \& MacIntyre, P. D. (2014). Capitalizing on individual differences: From premise to Practice. Bristol: Multilingual Matters.

Guiora, A. (1972). Construct validity and transpositional research: Toward an empirical study of psychoanalytic concepts. Comprehensive Psychiatry, 13(2), 139-150.

Hashimoto, Y. (2002). Motivation and willingness to communicate as predictors of reported L2 use: The Japanese ESL context. Second Language Studies, 20(2), 29-70.

Horwitz, E. K. (2013). Anxiety and Beliefs: On Learners, Teachers, and Pink Dresses. Invited presentation at the annual meeting of the International Association of Teachers of English to Speakers of Other Languages.

Horwitz, E. K. (2017). On the misreading of Horwitz, Horwitz, and Cope (1986) and the need to balance anxiety research and the experiences of anxious language learners. In C. Gkonou, M. Daubney \& J.-M. Dewaele (Eds.), New Insights into Language Anxiety: Theory, Research and Educational Implications (pp. 31-47). Bristol: Multilingual Matters.

Horwitz, E. K., Horwitz, M. \& Cope, J. A. (1986). Foreign language classroom anxiety. The Modern Language Journal, 70(2), 125-132.

Khazaei, Z. M., Zahed, A. M. \& Ketabi, S. (2012). Willingness to communicate in Iranian EFL learners: The effect of class size. English Language Teaching, 5(11), 181-187.

Kim, J.-H. (2000). Foreign Language Listening Anxiety: A Study of Korean Students Learning English. Unpublished doctoral dissertation, University of Texas, Austin, TX.

Lamb, M. (2004). Integrative motivation in a globalizing world. System, 32(1), 3-19.

Lee, J. H. (2018). The effects of short-term study abroad on L2 anxiety, international posture, and L2 willingness to communicate. Journal of Multilingual and Multicultural Development, 39(8), $703-714$.

Levis, J. M., Sonsaat, S., Link, S. \& Barriuso. T. A. (2016). Native and nonnative teachers of L2 pronunciation: Effects on learner performance. TESOL Quarterly, 50(4), 894-931. 
Lipovsky, C. \& Mahboob, A. (2010). Appraisal of native and non-native English speaking teachers. In A. Mahboob (Ed.), The NNEST lens: Nonnative English Speakers in TESOL (pp. 154-179). Newcastle upon Tyne: Cambridge Scholars.

Ma, L. P. F. (2012). Advantages and disadvantages of native-and nonnative-English-speaking teachers: Student perceptions in Hong Kong. TESOL Quarterly, 46(2), 280-305.

MacIntyre, P. D. (2017). An overview of language anxiety research and trends in its development. In C. Gkonou, M. Daubney \& J.-M. Dewaele (Eds.), New Insights into Language Anxiety: Theory, Research and Educational Implications (pp. 11-30). Bristol: Multilingual Matters.

MacIntyre, P. D., Clément, R., Dörnyei, Z. \& Noels, K. A. (1998). Conceptualizing willingness to communicate in a L2: a situational model of L2 confidence and affiliation. The Modern Language Journal, 82(4), 545-562.

MacIntyre, P. D. \& Legatto, J. J. (2011). A dynamic system approach to willingness to communicate: Developing an idiodynamic method to capture rapidly changing affect. Applied Linguistics, 32, 149-171.

Munro, M. J. \& Derwing, T. M. (1995). Foreign accent, comprehensibility, and intelligibility in the speech of second language learners. Language Learning, 45(1), 73-97.

Mystkowska-Wiertelak, A. \& Pawlak, M. (2017). Willingness to Communicate in Instructed Second Language Acquisition: Combining a Macro- and Micro-perspective. Bristol: Multilingual Matters.

Pekrun, R. (1992). Expectancy-value theory of anxiety: Overview and implications. In D. Forgays \& T. Sosnowski (Eds.), Anxiety: Recent Developments in Cognitive, Psychological and Health Research (pp. 23-39). Washington, DC: Hemisphere.

Peng, J. E. (2007). Willingness to communicate in the Chinese EFL classroom: A cultural perspective. In J. Liu (Ed.), English Language Teaching in China: New Approaches, Perspectives, and Standards (pp. 250-269). London: Continuum.

Peng, J. E. (2014). Willingness to Communicate inside the EFL Classroom: An Ecological Perspective. Bristol: Multilingual Matters.

Peng, J. E. \& Woodrow, L. (2010). Willingness to communicate in English: A model in the Chinese EFL classroom context. Language Learning, 60(4), 834-876.

Piechurska-Kuciel, E. (2008). Language Anxiety in Secondary Grammar School Students. Opole: Wydawnictwo Uniwersytetu Opolskiego (University of Opole Press).

Phillips, E. M. (1992). The effects of language anxiety on student's oral test performance and attitudes. The Modern Language Journal, 76(1), 14-26.

Rindal, U. (2010). Constructing identity with L2: Pronunciation and attitudes among Norwegian learners of English. Journal of Sociolinguistics, 14(2), 240-261.

Saito, Y., Garza, T. J. \& Horwitz, E. K. (1999). Foreign language reading anxiety. The Modern Language Journal, 83(2), 202-218.

(C) Servicio de Publicaciones. Universidad de Murcia. All rights reserved. IJES, vol. 21(1), 2021, pp. 93-113 Print ISSN: 1578-7044; Online ISSN: 1989-6131 
Savignon, S. J. (2005). Communicative Language Teaching: Strategies and Goals. In E. Hinkel (Ed.), Handbook of Research in Second Language Teaching and Learning (pp. 635-651). Mahwah, NJ: Lawrence Erlbaum.

Setter, J. (2008). Theories and Approaches in English Pronunciation. In R. Monroy \& A. Sánchez (Eds.), 25 Years of Applied Linguistics in Spain: Milestones and Challenges (pp. 447-457). Murcia: Universidad de Murcia.

Tang, G. \& Johnson, R. K. (1993). Implementing language change in Hong Kong schools: An ecological approach to evaluation. Journal of Asian Pacific Communication, 4(1), 31-47.

Thompson, A. S. \& Lee, J. (2014). The impact of experience abroad and language proficiency on language learning anxiety. TESOL Quarterly, 48(2), 252-274.

Tóth, Z. (2017). Exploring the relationship between anxiety and advanced Hungarian EFL learners' communication experiences in the target language: A study of high- vs low-Anxious learners. In C. Gkonou, M. Daubney \& J.-M. Dewaele (Eds.), New Insights into Language Anxiety: Theory, Research and Educational Implications (pp. 157-173). Bristol: Multilingual Matters.

Vasa, R. A. \& Pine, D. S. (2004). Neurobiology. In T. R. Morris \& J. S. March (Eds.), Anxiety Disorders in Children and Adolescents (pp. 3-26). New York: Guilford Press.

Walker, R. (2011). Teaching the Pronunciation of English as a Lingua Franca. Oxford: Oxford University Press.

Young, D. J. (1991). Creating a low-anxiety classroom environment: What does the language anxiety research suggest? The Modern Language Journal, 75, 425-439.

Zhang, J., Beckmann, N. \& Beckmann, J. F. (2018). To talk or not to talk: A review of situational antecedents of willingness to communicate in the second language classroom. System, 72, 226239.

(C) Servicio de Publicaciones. Universidad de Murcia. All rights reserved. IJES, vol. 21(1), 2021, pp. 93-113

Print ISSN: 1578-7044; Online ISSN: 1989-6131 